M. von Renteln

Nagoya Math. J.

Vol. 84 (1981), 1-8

\title{
BOUNDARY BEHAVIOR OF POSITIVE HARMONIC FUNCTIONS IN BALLS OF $\boldsymbol{R}^{n}$
}

\author{
MICHAEL VON RENTELN
}

\section{§1. Introduction}

Let $\boldsymbol{R}^{n}$ be the real $n$-dimensional euclidean space. Elements of $\boldsymbol{R}^{n}$ are denoted by $x=\left(x_{1}, \cdots, x_{n}\right)$, and $\|x\|$ denotes the euclidean norm of $x$. The open ball $B(x, r)$ with center $x$ and radius $r$ is defined by

$$
B(x, r):=\{y:\|y-x\|<r\}
$$

and the sphere $S(x, r)$ is defined by

$$
S(x, r):=\{y:\|y-x\|=r\} .
$$

In particular $B:=B(0,1)$ is the unit ball and $S:=S(0,1)$ is the unit sphere.

Let $u$ be a positive harmonic function in $B$. Then by the Herglotz Theorem ([2], p. 29) there exists a positive Borel measure $\mu$ on the unit sphere $S$ such that

$$
u(z)=\frac{1}{\sigma_{n}} \int_{S} P(z, x) d \mu(x) \quad(x \in S)
$$

holds for all $z \in B$. $P(z, x)$ is the Poisson kernel for $B$ defined by

$$
P(z, x):=\frac{1-\|z\|^{2}}{\|z-x\|^{n}}
$$

and $\sigma_{n}$ is the surface area of $S$.

Now the question arises of the relationship between the limiting behavior of $u(z)$ as $z$ approaches a boundary point and the measure $\mu$ on the boundary. To study this question we define the open polar cap $J(a, r)$ having center $a \in S$ and radius $r$ by

$$
J(a, r):=\{x \in S:\|x-a\|<r\}
$$

Received September 25, 1979. 
and the symmetric derivative $(D \mu)(a)$ of the measure $\mu$ at a surface point $a$ by

$$
(D \mu)(a):=\lim _{r \rightarrow 0} \frac{\mu}{m}[J(a, r)]
$$

where

$$
\frac{\mu}{m}[J(a, r)]:=\frac{\mu[J(a, r)]}{m[J(a, r)]}
$$

and $m$ is Lebesgue measure on the sphere. We note that $\gamma_{n}^{\prime} r^{n-1} \leqq m[J(a, r)] \leqq$ $\gamma_{n} r^{n-1}$ for $0 \leqq r<1$, where $\gamma_{n}, \gamma_{n}^{\prime}$ are constants depending only on the dimension $n$. The first main result regarding the limiting behavior of $u$ is the following, which is due to Fatou (1906) in case $n=2$ and to Bray and Evans (1927) for $n=3$.

Fatou's Theorem. Let $u$ be a positive harmonic function in the open unit ball of $\boldsymbol{R}^{n}$ having measure $\mu$ in its Herglotz representation. If $(D \mu)(a)$ exists, then the radial limit $\lim _{r \rightarrow 1} u(r a)$ exists and is equal to $(D \mu)(a)$.

Remark. The Theorem of Fatou also holds in the case $(D \mu)(a)=+\infty$. We indicate a short proof of this fact. Let $x \in J(a, 1-r)$. Using the triangle inequality we get

$$
\|r a-x\| \leqq\|r a-a\|+\|a-x\| \leqq 2(1-r) .
$$

From this it follows that

$$
\begin{aligned}
u(r a) & =\frac{1}{\sigma_{n}} \int_{S} \frac{1-r^{2}}{\|r a-x\|^{n}} d \mu(x) \geqq \frac{1}{\sigma_{n}} \int_{J(a, 1-r)} \frac{1-r}{2^{n}(1-r)^{n}} d \mu(x) \\
& =\frac{1}{2^{n} \sigma_{n}} \frac{\mu[J(a, 1-r)]}{(1-r)^{n-1}} \geqq \frac{\gamma_{n}^{\prime}}{2^{n} \sigma_{n}} \frac{\mu}{m}[J(a, 1-r)] \rightarrow+\infty
\end{aligned}
$$

for $r \rightarrow 1$, since $\left(D_{\mu}\right)(a)=+\infty$.

Fatou's Theorem also holds, if $z$ approaches the point $a$ in a nontangential manner (in a Stolz domain at a), see e.g. [2], p. 55. But if we replace Stolz domains by more general regions the situation changes dramatically. In this case the boundary behavior of $u$ can be very erratic. To see this, let us introduce regions $R(a, \delta, \gamma)$ in $B$ touching the unit sphere at $a$.

Definition. For $a \in S, \delta>0, \gamma \geqq 1$ let

$$
R(a, \delta, \gamma):=\left\{z \in B: 1-\|z\| \geqq \delta\left\|z^{\prime}-a\right\| r\right\} \backslash\{a\},
$$


where $z^{\prime}$ is the radial projection of $z$ on the sphere, i.e. $z^{\prime}=z /\|z\|$ if $z \neq 0$ and we define $z^{\prime}=a$ if $z=0$.

Evidently the radius $\{r a: 0 \leqq r<1\}$ lies in $R(a, \delta, \gamma)$ for any $\delta>0$, $\gamma \geqq 1$. If $\gamma=1$ the region $R(a, \delta, \gamma)$ is essentially a Stolz domain at $a$, i.e. a cone with vertex at $a$ and aperture depending on $\delta$. As $\gamma$ increases, $R(a, \delta, \gamma)$ touches the unit sphere with an increasing degree of tangency, e.g. for $\gamma=2$ and $n=2, R(a, \delta, \gamma)$ is essentially the interior of an oricycle at $a$. In two dimensions the regions $R(a, \delta, \gamma)$ were introduced by Cargo [1] to study tangential limits of Blaschke products.

\section{§2. The distinction between angular and tangential boundary behavior}

We will now make clear the difference between $\gamma=1$ and $\gamma>1$ regarding the boundary behavior of $u$. For simplicity we choose the dimension $n=2$, the boundary point $a=1$ and $\delta=1$. Let $\gamma>1$ be given. We construct a positive harmonic function $u$, such that $u\left(z_{n}\right) \rightarrow 0$ for every sequence $z_{n} \in R(a, \delta, 1), z_{n} \rightarrow a$, but for any number $c \geqq 0$ (including $c=$ $+\infty$ ) there exists a sequence $z_{n} \in R(a, \delta, \gamma), z_{n} \rightarrow a$ with $u\left(z_{n}\right) \rightarrow c$. In other words, the partial cluster sets of $u$ on $R(a, \delta, 1)$ or $R(a, \delta, \gamma)$ respectively consist of only one point 0 or is the whole interval $[0, \infty]$ respectively. One should note that the simple example of a harmonic function $h$ given in Helms ([2], p. 54) does not work here, since the partial cluster set of $h$ on $R(a, \delta, \gamma)$ consists of exactly one point in all cases $1<\gamma<2$.

Construction of the measure. We choose the discrete singular measure

$$
\mu=\sum_{k=1}^{\infty} s_{k} \delta_{\left(a_{k}\right)}
$$

with $a_{k}=\exp \left(i t_{k}\right), t_{k}=2^{-k}, s_{k}=t_{k}^{\beta}, 1<\beta<\gamma . \delta_{\left(a_{k}\right)}$ is the Dirac measure associated with the point $a_{k}$. Let $u$ be the positive harmonic function with this measure $\mu$ in its Herglotz representation.

1. Case. Let $0<r<\frac{1}{2}$, then there exists an index $n \in N$ with

$$
t_{n+1}<r \leqq t_{n} .
$$

This implies

$$
0 \leqq \frac{\mu}{m}[J(a, r)] \leqq \frac{1}{2 t_{n+1}} \mu\left[J\left(a, t_{n}\right)\right] .
$$

A short calculation yields 


$$
\frac{1}{2 t_{n+1}} \mu\left[J\left(a, t_{n}\right)\right]=\frac{1}{2 t_{n+1}} \sum_{k=n}^{\infty} s_{k}<2 t_{n}^{\beta-1} \rightarrow 0 \quad(n \rightarrow \infty)
$$

Therefore $(D \mu)(a)=0$ and it follows by Fatou's Theorem $u\left(z_{n}\right) \rightarrow 0$ for any sequence $z_{n} \in R(a, \delta, 1), z_{n} \rightarrow a$.

2. Case. We choose points $z_{n}=r_{n} e^{i t_{n}}$ with $1-r_{n}=t_{n}^{r}$. Thus $z_{n} \in$ $R(a, \delta, \gamma)$ for all $n \in N$. Let $I_{n}$ be the interval around $t_{n}$ defined by

$$
I_{n}:=\left\{e^{i t}:\left|t-t_{n}\right|<t_{n}^{r}\right\} \text {. }
$$

For $n$ sufficiently large, i.e. $n>(\gamma-1)^{-1}$, the only point $t_{k}$ belonging to $I_{n}$ is the point $t_{n}$. Therefore $\mu\left(I_{n}\right)=s_{n}$. By standard estimations we obtain

$$
\begin{aligned}
u\left(z_{n}\right) & \geqq \int_{S} \frac{1-r_{n}}{\left(1-r_{n}\right)^{2}+\left(t_{n}-t\right)^{2}} d \mu \geqq \int_{I_{n}} \frac{t_{n}^{r}}{t_{n}^{2 r}+\left(t_{n}-t\right)^{2}} d \mu \\
& \geqq \frac{1}{2 t_{n}^{r}} \int_{I_{n}} d \mu=\frac{1}{2} t_{n}^{\beta-\gamma} \rightarrow \infty \quad(n \rightarrow \infty) .
\end{aligned}
$$

Thus $u\left(z_{n}\right) \rightarrow \infty(n \rightarrow \infty)$.

For $n \in N$ let $A_{n}:=B(a, 1 / n) \cap R(a, \delta, \gamma)$. Since $u$ is continuous on $A_{n}$ and $A_{n}$ is a connected set, $u\left(A_{n}\right)$ is also connected. Since $u\left(A_{n}\right) \subset \boldsymbol{R}$ and the only connected subsets of $\boldsymbol{R}$ are the intervals, $u\left(A_{n}\right)$ must be an interval. From the construction above we see that $u\left(A_{n}\right)=(0, \infty)$ for every $n \in N$. Therefore the partial cluster set of $u$ on $R(a, \delta, \gamma)$ is $\cap_{n=1}^{\infty} \overline{u\left(A_{n}\right)}=[0, \infty]$

\section{§3. The problem}

Let $u$ be a positive harmonic function in $B$ represented by the measure $\mu$ on $S$ and let $a \in S$. We are interested in the behavior of $u$ in the region $R(a, \delta, \gamma)$. Of course, this depends on the measure $\mu$ on $S$ and especially on the behavior of $\mu$ in a neighbourhood of $a$. In view of the possibly erratic limiting behavior, our main problem is to give a condition on the measure $\mu$ such that $u$ is bounded in $R(a, \delta, \gamma)$. An obvious necessary condition is that $\mu$ be continuous at the point $a$, i.e. $\mu(\{a\})=0$, and an obvious sufficient condition is that there exist $r>0$ such that $\mu[J(a, r)]$ $=0$. But there are much weaker conditions, e.g. one can show that the condition

$$
\int_{S} \frac{d \mu(x)}{\|a-x\|^{r(n-1)}}<\infty
$$


is sufficient. Let us give an interpretation of this condition. Take the neighbourhood $J(a, r)$ of the point $a$. Then the condition says that $\mu[J(a, r)]$ tends to zero with some speed as $r \rightarrow 0$. It is not hard to show that the condition above is not necessary. In order to get a necessary and sufficient condition we introduce the following maximal function.

Definition. For a finite positive Borel measure $\mu$ on $S$ and a region $R=R(a, \delta, \gamma)$, we define the real function $M=M(R, \mu, x)$ on $S$ and the number $N=N(R, \mu)$ by

$$
\begin{aligned}
& M(R, \mu, x):=\sup \left\{\frac{\mu}{m}[J(x, r)]: r>\delta\|x-a\|^{r}\right\}, \\
& N(R, \mu):=\sup \left\{M\left(R, \mu, z^{\prime}\right): z \in R(a, \delta, \gamma)\right\},
\end{aligned}
$$

where $z^{\prime}$ is the radial projection of $z$ onto the sphere $S$ (with the agreement $z^{\prime}=a$ if $z=0$ ).

Remark. In the special case $n=2, \gamma=1$ and $\mu$ absolutely continuous, $M(R, \mu, x)$ is the Hardy-Littlewood maximal function.

Some auxiliary results will be given in the next section.

\section{§4. Auxiliary results}

Lemma 1. There exist positive constants $C_{1}$ and $C_{2}$, depending only on the dimension $n$, such that for all $z \in B, x \in S$ the Poisson kernel can be estimated as follows:

$$
C_{1} \frac{1-\|z\|}{(1-\|z\|)^{n}+\left\|x-z^{\prime}\right\|^{n}} \leqq P(z, x) \leqq C_{2} \frac{1-\|z\|}{(1-\|z\|)^{n}+\left\|x-z^{\prime}\right\|^{n}},
$$

where $z^{\prime}$ is the radial projection of $z$ onto $S$. In case of $z=0$ the inequality holds for any $z^{\prime} \in S$. One can choose $C_{1}=1 / n$ and $C_{2}=2 \cdot 3^{n}$.

Proof. We note that $\left\|z-z^{\prime}\right\|=1-\|z\|=\operatorname{dist}(z, S)$ and

$$
1-\|z\| \leqq\|x-z\| \text {. }
$$

The triangle inequality implies

$$
\|x-z\| \leqq\left\|x-z^{\prime}\right\|+(1-\|z\|)
$$

and

$$
\left\|x-z^{\prime}\right\| \leqq\|x-z\|+(1-\|z\|) \leqq 2\|x-z\| .
$$

Now (1), (2), (3) imply 


$$
\frac{1}{3}\left\{\left\|x-z^{\prime}\right\|+(1-\|z\|)\right\} \leqq\|x-z\| \leqq\left\{\left\|x-z^{\prime}\right\|+(1-\|z\|)\right\} .
$$

After exponentiating and further estimating we obtain

$$
\left(\frac{1}{8}\right)^{n}\left\{\left\|x-z^{\prime}\right\|^{n}+(1-\|z\|)^{n}\right\} \leqq\|x-z\|^{n} \leqq n\left\{\left\|x-z^{\prime}\right\|^{n}+(1-\|z\|)^{n}\right\},
$$

and from this the result follows.

Lemma 2. If $u$ is a positive harmonic function in $B$ with associated measure $\mu$ in the Herglotz representation, then

$$
u(z) \leqq C M\left(R, \mu, z^{\prime}\right)
$$

holds for all $z \in R(a, \delta, \gamma) . \quad C$ is a constant depending only on the dimension $n$.

Remark. In the special case of a Stolz domain, i.e. $\gamma=1$, and for $\mu$ absolutely continuous, the estimate in Lemma 2 is essentially known. See E. Stein ([3], p. 62, Theorem 1a) for an analogous $n$-dimensional statement.

Proof. Fix a point $z \in R(a, \delta, \gamma)$. We decompose the sphere $S$ in a union of subsets $S_{k}$ depending on $z$. Let $S_{k}=S_{k}(z)$ be defined as follows:

$$
\begin{aligned}
& S_{0}:=\left\{x \in S:\left\|x-z^{\prime}\right\|<1-\|z\|\right\}, \\
& S_{k}:=\left\{x \in S: 2^{k-1}(1-\|z\|) \leqq\left\|x-z^{\prime}\right\|<2^{k}(1-\|z\|)\right\},
\end{aligned}
$$

where $k=1,2, \cdots$. For $k=0,1,2, \cdots$ let $I_{k}:=\bigcup_{1=0}^{k} S_{1}$. We note that $I_{k}$ is the open polar cap $J\left[z^{\prime}, 2^{k}(1-\|z\|)\right.$ of radius $2^{k}(1-\|z\|)$. Let $p$ be the smallest integer $k$ with $2^{k}(1-\|z\|) \geqq 1$. We put $I_{p}=S$. Therefore,

$$
m\left(I_{k}\right) \leqq \gamma_{n} 2^{k(n-1)}(1-\|z\|)^{n-1} \quad(k<p) .
$$

Using Lemmas 1 and 2 we can estimate as follows:

$$
\begin{aligned}
\int_{S} P(z, x) d \mu(x) & \leqq C_{2} \int_{S} \frac{1-\|z\|}{(1-\|z\|)^{n}+\left\|x-z^{\prime}\right\|^{n}} d \mu(x) \\
& \leqq C_{2}\left\{\int_{S_{0}} \frac{1-\|z\|}{(1-\|z\|)^{n}} d \mu(x)+\sum_{k=1}^{P} \int_{S_{k}} \frac{1-\|z\|}{\left\|x-z^{\prime}\right\|^{n}} d \mu(x)\right\} \\
& \leqq C_{2}\left\{\frac{\mu\left(I_{0}\right)}{(1-\|z\|)^{n-1}}+\sum_{k=1}^{P} \frac{\mu\left(I_{k}\right)}{2^{n(k-1)}(1-\|z\|)^{n-1}}\right\} \\
& \leqq C_{2} \gamma_{n}\left\{\frac{\mu\left(I_{0}\right)}{m\left(I_{0}\right)}+\sum_{k=1}^{P} \frac{2^{n} \mu\left(I_{k}\right)}{2^{k} m\left(I_{k}\right)}\right\} \\
& \leqq C_{2} \gamma_{n}\left(1+2^{n} \sum_{k=1}^{P} \frac{1}{2^{k}}\right) \sup \left\{\frac{\mu}{m}\left[J\left(z^{\prime}, r\right)\right]: r>(1-\|z\|)\right\}
\end{aligned}
$$




$$
\leqq C_{2} \gamma_{n}\left(1+2^{n}\right) M\left(R, \mu, z^{\prime}\right)
$$

This yields $u(z) \leqq C M\left(R, \mu, z^{\prime}\right)$ with $C=C_{2} \gamma_{n}\left(1+2^{n}\right) / \sigma_{n}$. Since the constant $C$ is independent of the special point $z \in R(a, \delta, \gamma)$, the inequality holds for all $z \in R(a, \delta, \gamma)$.

\section{§. The main result}

TheOREM. A positive harmonic function $u$ in $B$ with measure $\mu$ in its Herglotz representation is bounded in the region $R(a, \delta, \gamma)$ if and only if $N(R, \mu)<\infty$.

Proof. One direction follows from Lemma 1. To prove the converse, assume that $u$ is bounded in $R(a, \delta, \gamma)$. Then there exists an absolute constant $M$, such that

$$
\int_{S} P(z, x) d \mu(x) \leqq M
$$

holds for all $z \in R(a, \delta, \gamma)$. To prove $N(R, \mu)<\infty$ we have to show that there exists an absolute constant $C$ such that

$$
\frac{\mu}{m}\left[J\left(z^{\prime}, r\right)\right] \leqq C
$$

holds for all $z \in R(a, \delta, \gamma)$ and all $r>\delta\left\|z^{\prime}-a\right\|^{r}$. It is clear that we may assume $r<1$. Let such a pair $z, r$ be given. We choose a special point $z_{r}=(1-r) z^{\prime}$. It follows that $\left\|z_{r}\right\|=1-r<1$, i.e. $z \in B$ and $1-\left\|z_{r}\right\|=$ $r$. Since $r>\delta\left\|z^{\prime}-a\right\|^{\gamma}$ we have $z_{r} \in R(a, \delta, \gamma)$, i.e. $1-\left\|z_{r}\right\| \geqq \delta\left\|z^{\prime}-a\right\|^{r}$. Using our assumption (1) for the point $z_{r}$ and Lemma 1 we obtain

$$
\begin{aligned}
M & \geqq \int_{S} P\left(z_{r}, x\right) d \mu(x) \geqq C_{1} \int_{S} \frac{1-\left\|z_{r}\right\|}{\left(1-\left\|z_{r}\right\|\right)^{n}+\left\|x-z_{r}^{\prime}\right\|^{n}} d \mu(x) \\
& \geqq C_{1} \int_{\left\|x-z_{r}^{\prime}\right\|<r} \frac{r}{r^{n}+r^{n}} d \mu(x)=\frac{1}{2} \frac{C_{1}}{r^{n-1}} \mu\left[J\left(z_{r}^{\prime}, r\right)\right] \\
& \geqq \frac{1}{2} C_{1} \gamma_{n}^{\prime} \frac{\mu}{m}\left[J\left(z^{\prime}, r\right)\right] .
\end{aligned}
$$

Note that $z^{\prime}=z_{r}^{\prime}$. Thus we have

$$
\frac{\mu}{m}\left[J\left(z^{\prime}, r\right)\right] \leqq C
$$

with the constant $C=2 M / C_{1} \gamma_{n}^{\prime}$, which is independent of $z$ and $r$. Therefore we have established (2). 


\section{REFERENCES}

[1] G. T. Cargo, Angular and tangential limits of Blaschke products and their successive derivatives, Can. J. Math., 14 (1962), 334-348.

[2] L. L. Helms, Introduction to potential theory, Wiley-Interscience, New York, 1969.

[ 3 ] E. M. Stein, Singular integrals and differentiability properties of functions, Princeton University Press, Princeton, 1970.

Mathematisches Institut der Universität

D-6300 Giessen, West-Germany

Current address:

Mathematische Institut I der Universität

D-7500 Karlsruhe, West-Germany 